\title{
Passive noninteracting control for human-robot team interaction
}

\author{
Selma Musić and Sandra Hirche
}

\begin{abstract}
This paper is motivated by the control of robot teams by a human. Control challenges arise because i) typically, the team needs to achieve multiple control objectives, shared between the robot team and the human, in order to accomplish a task, ii) robust stability needs to be guaranteed to facilitate the safe interaction with the human and the apriori unknown environment. The concept of passivity has been successfully applied for robust stabilization of robotic systems, however, not in the context of shared control in human-robot team interaction. In this paper we propose a novel control approach which decouples the robot team dynamics into multiple subsystems, each having a different control objective. The proposed control law, suitable for the interaction of the robot team with the human or environment, guarantees passivity of the subsystems. The approach is illustrated in a simulation.
\end{abstract}

\section{INTRODUCTION}

The control of complex dynamical systems with the human in the loop and interaction with the environment is relevant in many application domains, e.g. process control, flight control, and human-robot team interaction [1], [2], to name just a few. Typically, multiple control objectives are defined for such systems in order to accomplish an overall task. Due to system complexity (high number of degrees of freedom) and cognitive limitations of the human, the operator cannot control all the system variables toward accomplishing the control objectives. In consequence, the human can only take over a limited number of tasks to control, while the remaining control tasks are taken over by autonomous functions. Therefore, control sharing between the human and the robot autonomy is required.

Control sharing determines the level of collaboration between the human operator and the (semi-)autonomous system, necessary to accomplish a task [3]. One of the approaches to the control sharing is to complement the humanin-the-loop control with autonomous functionalities [4]. In this way, multiple control goals can be achieved, often simultaneously. In order to obtain complementary shared control, it is necessary to ensure noninteraction (decoupling) of the control loops, so that reference inputs of one control loop do not have undesired effects on states and outputs of the other control loops. Noninteracting (Decoupling) control of MIMO nonlinear, affine systems through state-feedback control is proposed in [5]. The proposed control approach decouples the system into SISO subsystems. A more general case, the decoupling into MIMO subsystems (blockpartitioned noninteraction) is analyzed in [6]. However, the

All authors are with the Chair of Information-oriented Control, Department of Electrical Engineering and Information Technology, Technical University of Munich, Munich, Germany. e-mail: \{selma.music, hirche\}@etum. de control design in this case is not straightforward, and only the properties of the general control law are derived.

In robotics, the decoupling control is applied on robot teams to enable the control of elementary behaviors. For example, elementary behaviors can represent velocity profiles of individual robots that, when combined, generate a complex team behavior [7]. However, the proposed approaches typically consider a simplified robot model (single integrator), see e.g. [8] and [9]. The Euler-Lagrange dynamics is taken into account in [10] and [11], where the team behaviors are designed to be decoupled. The noninteracting control is applied in [12] on a linearized model of dual manipulators, constrained by an object. However, generalizing the approach to multiple and arbitrary behaviors for Euler-Lagrange dynamics is not considered so far. Guaranteeing passivity of the closed-loop system implies safety of interactions [13]. Therefore, it is important for the human-in-the-loop interaction, e.g. teleoperation through a haptic device [14], direct physical interaction, close-range teleoperation [2], and interactions of the robots with environment [15]. Passivity also relates to the classical approaches of analyzing stability of dynamical systems, e.g. Lyapunov theory and $L_{2}$ stability [16] and can be used to analyze stability of human-robot team control loops.

In this paper we propose an explicit, block-partitioned noninteracting control approach for Lagrangian systems. It allows the decoupling of the system into multiple subsystems with each subsystem having a different control objective. This is accomplished by introducing the concept of subtasks. The novelty of this paper is the solution of the blockpartitioned noninteracting control with passivity guarantees for Lagrangian systems, which is achieved by preserving Lagrangian dynamical properties after the system decoupling. Our approach is applicable not only to robot teams in free motion but also to robot teams with physical coupling as it occurs in cooperative object manipulation for example. It ultimately allows the safe control sharing in human-robot team interaction.

The paper is outlined as follows: in Section II the problem is formally posed. The block-partitioned noninteracting control for Lagrangian systems is proposed in Section III, where the control design is split into two stages: input-output and state noninteraction. The passivity guarantees are presented in Section IV. Numerical example and simulation results are reported in Section V, and conclusion is provided in Section VI.

Notations: Vectors are represented by lower-case, bold letters, while matrices are represented by capital, bold letters. The Lie derivative of a function $h(\boldsymbol{x})$ along a vector field 
$\boldsymbol{f}(\boldsymbol{x})$ is given as $\mathcal{L}_{\boldsymbol{f}} h(\boldsymbol{x})=\frac{\partial h(\boldsymbol{x})}{\partial \boldsymbol{x}} \boldsymbol{f}(\boldsymbol{x})$. Distributions are represented by calligraphic letters, e.g. $\mathcal{D}$, and their codistributions by $\mathcal{D}^{\perp}$. The differential of a function $f$ is denoted as $\mathrm{d} f$.

\section{PROBLEM STATEMENT}

The dynamics of each robot is given by the Euler-Lagrange equation

$$
\boldsymbol{M}_{i}\left(\boldsymbol{q}_{i}\right) \ddot{\boldsymbol{q}}_{i}+\boldsymbol{C}_{i}\left(\boldsymbol{q}_{i}, \dot{\boldsymbol{q}}_{i}\right) \dot{\boldsymbol{q}}_{i}+\boldsymbol{\tau}_{i}^{g}\left(\boldsymbol{q}_{i}\right)=\boldsymbol{\tau}_{i}^{c}+\boldsymbol{\tau}_{i}^{e},
$$

where $\boldsymbol{q}_{i} \in \mathcal{Q}_{i}$ is the $n$-dimensional vector of generalized coordinates, and $\dot{\boldsymbol{q}}_{i}, \ddot{\boldsymbol{q}}_{i}$ are the corresponding velocity and acceleration vectors, respectively. Inertia and Coriolis and centrifugal $n \times n$ matrices are given by $\boldsymbol{M}_{i}\left(\boldsymbol{q}_{i}\right)$ and $\boldsymbol{C}_{i}\left(\boldsymbol{q}_{i}, \dot{\boldsymbol{q}}_{i}\right)$, respectively. Vectors $\boldsymbol{\tau}_{i}^{g}\left(\boldsymbol{q}_{i}\right), \boldsymbol{\tau}_{i}^{c}$, and $\boldsymbol{\tau}_{i}^{e}$ are $n$-dimensional gravitation, control, and external torque terms, respectively. Inertia matrix is positive-definite and symmetric, and $\dot{\boldsymbol{M}}_{i}\left(\boldsymbol{q}_{i}\right)-2 \boldsymbol{C}_{i}(\boldsymbol{q}, \dot{\boldsymbol{q}})$ is skew-symmetric. The vector of external torques, $\boldsymbol{\tau}_{i}^{e}$, can contain physical couplings between the robots within the team, e.g. as it arises in cooperative object manipulation or torques arising from the interaction with the human.

Let us assume the multi-robot system consists of $N$ robots, each described by (1). Then the robot team dynamics can be described on $n \times N$ product manifold $\mathcal{Q}=\mathcal{Q}_{1} \times \ldots \times \mathcal{Q}_{N}$ as

$$
\boldsymbol{M}(\boldsymbol{q}) \ddot{\boldsymbol{q}}+\boldsymbol{C}(\boldsymbol{q}, \dot{\boldsymbol{q}}) \dot{\boldsymbol{q}}+\boldsymbol{\tau}^{g}(\boldsymbol{q})=\boldsymbol{\tau}^{c}+\boldsymbol{\tau}^{e},
$$

where $\boldsymbol{q}=\left[\boldsymbol{q}_{1}^{\top}, \ldots, \boldsymbol{q}_{N}^{\top}\right]^{\top}, \boldsymbol{M}=\operatorname{blkdiag}\left[\boldsymbol{M}_{1}, \ldots, \boldsymbol{M}_{N}\right]$, $\boldsymbol{C}=\operatorname{blkdiag}\left[\boldsymbol{C}_{1}, \ldots, \boldsymbol{C}_{N}\right], \boldsymbol{\tau}^{g}=\left[\left(\boldsymbol{\tau}_{1}^{g}\right)^{\top}, \ldots,\left(\boldsymbol{\tau}_{N}^{g}\right)^{\top}\right]^{\top}, \boldsymbol{\tau}^{c}=$ $\left[\left(\boldsymbol{\tau}_{1}^{c}\right)^{\top}, \ldots,\left(\boldsymbol{\tau}_{N}^{c}\right)^{\top}\right]^{\top}$, and $\boldsymbol{\tau}^{e}=\left[\left(\boldsymbol{\tau}_{1}^{e}\right)^{\top}, \ldots,\left(\boldsymbol{\tau}_{N}^{e}\right)^{\top}\right]^{\top}$.

Let us cast the system dynamics (2) into an affine, statespace form

$$
\left[\begin{array}{c}
\dot{\boldsymbol{q}} \\
\ddot{\boldsymbol{q}}
\end{array}\right]=\underbrace{\left[\begin{array}{c}
\dot{\boldsymbol{q}} \\
-\boldsymbol{M}^{-1}\left(\boldsymbol{C} \dot{\boldsymbol{q}}+\boldsymbol{\tau}^{g}\right)
\end{array}\right]}_{\boldsymbol{f}(\boldsymbol{q}, \dot{\boldsymbol{q}})}+\underbrace{\left[\begin{array}{c}
\mathbf{0} \\
\boldsymbol{M}^{-1}
\end{array}\right]}_{\boldsymbol{G}(\boldsymbol{q})}\left(\boldsymbol{\tau}^{c}+\boldsymbol{\tau}^{e}\right),
$$

where $\boldsymbol{f}=\left[\boldsymbol{f}_{1}^{\top}, \boldsymbol{f}_{2}^{\top}\right]^{\top}, \boldsymbol{G}=\left[\boldsymbol{G}_{1}^{\top}, \boldsymbol{G}_{2}^{\top}\right]^{\top}=\left[\boldsymbol{g}_{1}, \ldots, \boldsymbol{g}_{n}\right]$, and the state dependency of the parameters is omitted for brevity.

Let us define the concept of a subtask which is important in our noninteracting control design.

Definition 1: A subtask $i$ is a vector $\left[\boldsymbol{x}_{s, i}^{\top}, \dot{\boldsymbol{x}}_{s, i}^{\top}\right]^{\top}$. The vector $\boldsymbol{x}_{s, i}$ is a smooth submersion, projection mapping of the state manifold $\mathcal{Q}$ onto a manifold $\mathcal{Q}_{s, i}$

$$
\phi_{i}: \mathcal{Q} \rightarrow \mathcal{Q}_{s, i}, \quad \boldsymbol{x}_{s, i}=\phi_{i}(\boldsymbol{q}) .
$$

The vector $\dot{\boldsymbol{x}}_{s, i}$ is the differential map of $\boldsymbol{x}_{s, i}$

$$
D \phi_{i}: T \mathcal{Q} \rightarrow T \mathcal{Q}_{s, i},
$$

that evolves on $T \mathcal{Q}_{s, i}$ and is surjective.

According to Definition 1, subtasks are projections of robot team states so that $\operatorname{dim}(\mathcal{Q}) \geq \operatorname{dim}\left(\mathcal{Q}_{s, i}\right)$. They impose equality constraints onto the system (3), which enables the definition of the system outputs in the following way

$$
\boldsymbol{y}=\left[\begin{array}{c}
\boldsymbol{y}_{1} \\
\vdots \\
\boldsymbol{y}_{p}
\end{array}\right]=\left[\begin{array}{c}
\boldsymbol{h}_{1}(\boldsymbol{q}, \dot{\boldsymbol{q}}) \\
\vdots \\
\boldsymbol{h}_{p}(\boldsymbol{q}, \dot{\boldsymbol{q}})
\end{array}\right]=\underbrace{\left[\begin{array}{c}
\frac{\partial \phi_{1}(\boldsymbol{q})}{\partial \boldsymbol{q}} \\
\vdots \\
\frac{\partial \phi_{\boldsymbol{p}}(\boldsymbol{q})}{\partial \boldsymbol{q}}
\end{array}\right]}_{\boldsymbol{J}_{s}(\boldsymbol{q})} \dot{\boldsymbol{q}}
$$

where $\boldsymbol{h}_{i}(\boldsymbol{q}, \dot{\boldsymbol{q}})=\left[h_{i, 1}(\boldsymbol{q}, \dot{\boldsymbol{q}}), \ldots, h_{i, p_{i}}(\boldsymbol{q}, \dot{\boldsymbol{q}})\right]^{\top}, i=1, \ldots, p$ is the mapping (5) and $p$ is the overall number of subtasks. Matrix $\boldsymbol{J}_{s}(\boldsymbol{q})=\left[\boldsymbol{J}_{s, 1}^{\top}(\boldsymbol{q}) ; \ldots ; \boldsymbol{J}_{s, p}^{\top}(\boldsymbol{q})\right]^{\top}$ is the task Jacobian, while $\boldsymbol{J}_{s, i}, \forall i=1, . ., p$ is the $i^{\text {th }}$ subtask Jacobian.

Example 1: For the task of object transportation by a team of robot manipulators, possible subtasks may be: grasping the object, nonviolation of constraints imposed by the object geometry (i.e. maintaining a formation), motion of the robot team in formation to the goal, inter-robot collision avoidance, obstacle avoidance, etc.

We focus our analysis on the Lagrangian systems with certain structural properties, outlined in the following assumptions.

Assumption 1: The system (3), (6) has no internal dynamics.

This assumption implies that there are no states in (3) which are unobservable. From the robotics point of view we restrict the analysis to the systems with no external rigid constraints, redundancy with respect to the task, or elastic structures [17].

Assumption 2: The sum of the number of outputs equals the total number of generalized coordinates, $\boldsymbol{q}$, i.e. $\sum_{i=1}^{p} p_{i}=n N$.

The condition $\sum_{i=1}^{p} p_{i}<n N$ implies the existence of the internal dynamics, which is excluded by Assumption 1. The condition $\sum_{i=1}^{p} p_{i}>n N$ is not considered since in this case prioritization of subtasks needs to be considered, which is left for future work.

Assumption 3: The subtask Jacobian $\boldsymbol{J}_{s}$, defined in (6), has a full rank.

We pose following problems to be solved in the remainder of the paper:

Problem 1: Design a control law

$$
\boldsymbol{\tau}^{c}=\boldsymbol{\alpha}(\boldsymbol{q}, \dot{\boldsymbol{q}})+\boldsymbol{\Gamma}(\boldsymbol{q}) \tilde{\boldsymbol{\tau}}^{c},
$$

with $\boldsymbol{\alpha}(\boldsymbol{q}, \dot{\boldsymbol{q}})$ being $n$-dimensional smooth vector field, and $\boldsymbol{\Gamma}(\boldsymbol{q})$ being a nonsingular, $n \times n$ dimensional matrix, which decouples the system (3), (6) into $p$ subsystems, so that for each $i=1, \ldots, p$, input block $\tilde{\boldsymbol{\tau}}_{i}^{c}$ affects only the output block $\boldsymbol{y}_{i}$. This means that for all $i \neq j$ the output $\boldsymbol{y}_{i}$ needs to be invariant under the input $\tilde{\boldsymbol{\tau}}_{j}^{c}$.

Problem 2: Design a control law $\tilde{\tau}^{c}$, for the $p$ obtained subsystems so that each subsystem is passive from the input $\tilde{\boldsymbol{\tau}}_{i}^{e}$ to the output $\boldsymbol{y}_{i}$.

Remark 1: In the context of human-robot team interaction, each input block $\tilde{\boldsymbol{\tau}}_{i}^{c}$ represents input channels from the human operator or from the autonomous planner(s). The 
dimensionality and type of the control inputs provided by the human depend on the interface through which the human interacts with the system, e.g. through a haptic device [10].

\section{NONINTERACTING CONTROL}

In this section we solve Problem $l$ by introducing noninteracting control for Euler-Lagrangian systems. First, we solve the input-output noninteracting control problem through the matrix $\boldsymbol{\Gamma}(\boldsymbol{q})$ in (7). Then, we solve the state noninteracting control problem through the vector $\boldsymbol{\alpha}(\boldsymbol{q}, \dot{\boldsymbol{q}})$ in (7) and a suitable coordinate transformation.

\section{A. Noninteracting input-output control}

In order to ensure that the specific inputs affect only the specific outputs, it is necessary to render the input-output behavior of robot dynamics, modeled by (3) and (6), noninteractive.

Let us insert (7) into (3), (6) to obtain

$$
\begin{aligned}
& {\left[\begin{array}{c}
\dot{\boldsymbol{q}} \\
\ddot{\boldsymbol{q}}
\end{array}\right]=\left[\begin{array}{c}
\boldsymbol{f}_{1}(\dot{\boldsymbol{q}}) \\
\tilde{\boldsymbol{f}}_{2}(\boldsymbol{q}, \dot{\boldsymbol{q}})
\end{array}\right]+\left[\begin{array}{c}
\mathbf{0} \\
\tilde{\boldsymbol{G}}_{2}(\boldsymbol{q})
\end{array}\right] \tilde{\boldsymbol{\tau}}^{c}+\boldsymbol{G}(\boldsymbol{q}) \boldsymbol{\tau}^{e}} \\
& {\left[\begin{array}{c}
\boldsymbol{y}_{1} \\
\vdots \\
\boldsymbol{y}_{p}
\end{array}\right]=\left[\begin{array}{c}
\boldsymbol{h}_{1}(\boldsymbol{q}, \dot{\boldsymbol{q}}) \\
\vdots \\
\boldsymbol{h}_{p}(\boldsymbol{q}, \dot{\boldsymbol{q}})
\end{array}\right]}
\end{aligned}
$$

where $\tilde{\boldsymbol{f}}_{2}(\boldsymbol{q}, \dot{\boldsymbol{q}})=\boldsymbol{f}_{2}(\boldsymbol{q}, \dot{\boldsymbol{q}})+\boldsymbol{G}_{2}(\boldsymbol{q}) \boldsymbol{\alpha}(\boldsymbol{q}, \dot{\boldsymbol{q}})$, and $\tilde{\boldsymbol{G}}_{2}(\boldsymbol{q})=$ $\boldsymbol{G}_{2}(\boldsymbol{q}) \boldsymbol{\Gamma}(\boldsymbol{q})=\left[\tilde{\boldsymbol{g}}_{1}(\boldsymbol{q}), \ldots, \tilde{\boldsymbol{g}}_{p}(\boldsymbol{q})\right]$.

A sufficient condition for achieving the input-output noninteraction is formulated below.

Proposition 1 ([18]): Consider the system (8) and suppose that the output $\boldsymbol{y}_{i}$ is invariant under the input $\tilde{\boldsymbol{\tau}}_{j}^{c}$, where $i \neq j$. Then

$$
\begin{aligned}
& \mathcal{L}_{\tilde{g}_{j}} \boldsymbol{h}_{i}(\boldsymbol{q}, \dot{\boldsymbol{q}})=\mathbf{0}, \\
& \mathcal{L}_{\tilde{\boldsymbol{g}}_{j}} \mathcal{L}_{\boldsymbol{z}_{1}} \ldots \mathcal{L}_{\boldsymbol{z}_{k}} \boldsymbol{h}_{i}(\boldsymbol{q}, \dot{\boldsymbol{q}})=\mathbf{0},
\end{aligned}
$$

for all $\boldsymbol{q}$ and $\dot{\boldsymbol{q}}$ and any combination of vector fields $\boldsymbol{z}_{1}, \ldots, \boldsymbol{z}_{k}$ from the set $\left\{\tilde{\boldsymbol{f}}_{2}, \tilde{\boldsymbol{g}}_{1}, \ldots, \tilde{\boldsymbol{g}}_{m}\right\}$.

Noninteracting control is achieved by ensuring that the inputs, which should not affect a particular output block, have a control effect in its kernel. Let us define the following kernel distributions

$$
\begin{aligned}
& \mathcal{K}_{i}=\operatorname{ker} \boldsymbol{J}_{s, i}=\operatorname{ker} \mathrm{d} \boldsymbol{h}_{i}=\bigcap_{j=1}^{p_{i}} \operatorname{ker} \mathrm{d} h_{i j}, \quad \forall i=1, \ldots, p, \\
& \mathcal{K}=\bigcap_{i=1}^{p} \mathcal{K}_{i},
\end{aligned}
$$

where $\mathcal{K}_{i}$ is the kernel distribution of the output block $\boldsymbol{y}_{i}$ and $\mathcal{K}$ is the intersection of all thus obtained kernel distributions. Furthermore, let us introduce controllability distributions relevant for solving the input-output noninteracting problem

$$
\begin{aligned}
& \tilde{\mathcal{D}}_{i}=\left\langle\tilde{\boldsymbol{f}}_{2}, \tilde{\boldsymbol{g}}_{1}, \ldots, \tilde{\boldsymbol{g}}_{p} \mid \operatorname{span}\left\{\tilde{\boldsymbol{g}}_{i}\right\}\right\rangle, \quad \forall i=1, \ldots, p, \\
& \tilde{\mathcal{D}}_{i}^{\star}=\left\langle\tilde{\boldsymbol{f}}_{2}, \tilde{\boldsymbol{g}}_{1}, \ldots, \tilde{\boldsymbol{g}}_{p} \mid \operatorname{span}\left\{\tilde{\boldsymbol{g}}_{j}: j \neq i\right\}\right\rangle, \quad \forall i=1, \ldots, p, \\
& \tilde{\mathcal{D}}^{\star}=\bigcap_{i=1}^{p} \tilde{\mathcal{D}}_{i}^{\star}, \\
& \tilde{\mathcal{D}}=\left\langle\tilde{\boldsymbol{f}}_{2}, \tilde{\boldsymbol{g}}_{1}, \ldots, \tilde{\boldsymbol{g}}_{p} \mid \operatorname{span}\left\{\tilde{\boldsymbol{g}}_{i}: i=1, \ldots, p\right\}\right\rangle,
\end{aligned}
$$

which are invariant under the vector fields $\left\{\tilde{\boldsymbol{f}}_{2}, \tilde{\boldsymbol{g}}_{1}, \ldots, \tilde{\boldsymbol{g}}_{p}\right\}$. $\tilde{\mathcal{D}}_{i}$ spans block $\tilde{\boldsymbol{g}}_{i}$ of $\tilde{\boldsymbol{G}}_{2}$, while $\tilde{\mathcal{D}}_{i}^{\star}$ spans all the blocks $\tilde{\boldsymbol{g}}_{j}$ such that $j \neq i$. $\tilde{\mathcal{D}}^{\star}$ is the intersection of all $\tilde{\mathcal{D}}_{i}^{\star}$ distributions and $\tilde{\mathcal{D}}$ spans $\tilde{G}_{2}$. The following equalities can be derived for the distributions in (11)

$$
\tilde{\mathcal{D}}_{i}^{\star}=\sum_{j \neq i} \tilde{\mathcal{D}}_{j}, \tilde{\mathcal{D}}=\sum_{i=1}^{p} \tilde{\mathcal{D}}_{i} \text { and } \tilde{\mathcal{D}}=\tilde{\mathcal{D}}_{i}^{\star}+\bigcap_{j \neq i} \tilde{\mathcal{D}}_{j}^{\star} .
$$

Equivalent distributions to (11) for the open loop system (3) are denoted as $\mathcal{D}_{i}, \mathcal{D}_{i}^{\star}, \mathcal{D}^{\star}$, and $\mathcal{D}$, respectively.

Condition (9) can now be expressed as follows

$$
\tilde{\mathcal{D}}_{j} \subset \bigcap_{i \neq j} \mathcal{K}_{i} \text { or } \tilde{\mathcal{D}}_{i}^{\star} \subset \mathcal{K}_{i}, \text { and } \tilde{\mathcal{D}}^{\star} \subset \mathcal{K},
$$

which means that it is necessary to find maximal, controlled invariant distributions $\tilde{\mathcal{D}}_{i}^{\star}$ (or $\tilde{\mathcal{D}}_{j}$ ) and $\tilde{\mathcal{D}}^{\star}$ which lie in the defined kernel spaces.

Let us now derive the properties of distributions in (11) for Lagrangian systems. Since the mapping (4) is smooth according to Definition 1, under Assumption 3 the codistributions defined by

$$
\mathcal{C}_{i}=\sum_{j=1}^{p_{i}} \operatorname{span}\left\{\mathrm{d} h_{i, j}\right\}, \quad \forall i=1, \ldots, p
$$

are nonsingular with $\operatorname{dim}\left\{\mathcal{C}_{i}\right\}=p_{i}$. It is possible to find distributions $\tilde{\mathcal{D}}_{i}^{\star}$ which lie in $\mathcal{K}_{i}$ according to the following algorithm [5]

$$
\begin{aligned}
& \Omega_{i_{0}}=\mathcal{C}_{i} \\
& \Omega_{i_{k}}=\Omega_{i_{k-1}}+\mathcal{L}_{\boldsymbol{f}_{2}}\left(\Omega_{i_{k-1}} \cap \mathcal{G}^{\perp}\right)+\sum_{i=1}^{m} \mathcal{L}_{\boldsymbol{g}_{i}}\left(\Omega_{i_{k-1}} \cap \mathcal{G}^{\perp}\right),
\end{aligned}
$$

where $\mathcal{G}=\operatorname{span}\left\{\boldsymbol{G}_{2}\right\}=\operatorname{span}\left\{\boldsymbol{g}_{1}, \ldots, \boldsymbol{g}_{p}\right\}$. If $\Omega_{i_{k+1}}=$ $\Omega_{i_{k}}$, the solution is reached and $\tilde{\mathcal{D}}_{i}^{\star}=\Omega_{i_{k}}^{\perp}$. Codistribution $\left(\Omega_{i_{k-1}} \cap \mathcal{G}^{\perp}\right)$ is trivial in our case, since $\operatorname{dim}\left\{\mathcal{G}^{\perp}\right\}=0$ as the matrix $\boldsymbol{G}_{2}(\boldsymbol{q})$ is a full rank, square matrix. Therefore, we conclude

$$
\tilde{\mathcal{D}}_{i}^{\star}=\mathcal{C}_{i}^{\perp} .
$$

Lemma 1: Consider the dynamical system (3), (6) under Assumptions 1-2. Then the distributions $\tilde{\mathcal{D}}, \tilde{\mathcal{D}}^{\star}, \tilde{\mathcal{D}}_{i}, \tilde{\mathcal{D}}_{i}^{\star}, \forall i=$ $1, \ldots, p$ are nonsingular with $\operatorname{dim}\{\tilde{\mathcal{D}}\}=n, \operatorname{dim}\left\{\tilde{\mathcal{D}}^{\star}\right\}=0$, $\operatorname{dim}\left\{\tilde{\mathcal{D}}_{i}\right\}=p_{i}$, and $\operatorname{dim}\left\{\tilde{\mathcal{D}}_{i}^{\star}\right\}=n-p_{i}$.

Proof: Since the inertial matrix $\boldsymbol{M}(\boldsymbol{q})$ of (3) is fullrank, the system satisfies the strong accessibility rank condition at each point $\boldsymbol{q}_{0} \in \mathcal{Q}$. Therefore, $\operatorname{dim}\{\mathcal{D}\}=n$ as it spans $\boldsymbol{G}$. Under the static control law (7) the accessibility of 
the closed-loop system does not change [18], which means that $\operatorname{dim}\{\tilde{\mathcal{D}}\}=\operatorname{dim}\{\mathcal{D}\}=n$. Considering the equality for $\tilde{\mathcal{D}}^{\star}$ in (11) and (15), by duality

$$
\left(\tilde{\mathcal{D}}^{\star}\right)^{\perp}=\sum_{i=1}^{p} \sum_{j=1}^{p_{i}} \mathrm{~d} h_{i, j},
$$

and $\operatorname{dim}\left\{\left(\tilde{\mathcal{D}}^{\star}\right)^{\perp}\right\}=n$ due to Assumption 2. Therefore, $\operatorname{dim}\left\{\left(\tilde{\mathcal{D}}^{\star}\right)\right\}=0$. Since $\operatorname{dim}\left\{\mathcal{C}_{i}\right\}=p_{i}$ and (15) holds, it follows that $\operatorname{dim}\left\{\tilde{\mathcal{D}}_{i}^{\star}\right\}=n-p_{i}$. Lastly, since $\tilde{\mathcal{D}}_{i}^{\star}+\tilde{\mathcal{D}}_{i}=\tilde{\mathcal{D}}$, it follows that $\operatorname{dim}\left\{\tilde{\mathcal{D}}_{i}\right\}=p_{i}$.

Now we are ready to state the suitable input-output decoupling matrix $\boldsymbol{\Gamma}(\boldsymbol{q})$.

Theorem 1: Consider the dynamical system (3), (6) under Assumptions 1-3. Then the input-output noninteracting control problem is solvable with the invertible matrix $\boldsymbol{\Gamma}(\boldsymbol{q})$, being the solution of

$$
\boldsymbol{J}_{s}(\boldsymbol{q}) \boldsymbol{G}_{2}(\boldsymbol{q}) \boldsymbol{\Gamma}(\boldsymbol{q})=\tilde{\boldsymbol{G}}_{s}(\boldsymbol{q})
$$

where $\tilde{\boldsymbol{G}}_{s}(\boldsymbol{q})=\operatorname{blkdiag}\left[\boldsymbol{M}_{s, 1}^{-1}(\boldsymbol{q}), \ldots, \boldsymbol{M}_{s, p}^{-1}(\boldsymbol{q})\right]$ and $M_{s, i}, \forall i=1, \ldots, p$ being positive-definite matrices.

Proof: The goal of the input-output noninteracting control law is to ensure that the obtained $\tilde{\boldsymbol{G}}_{s}(\boldsymbol{q})$ is block diagonal. Let us write out (16) as

$$
\left[\begin{array}{ccc}
\boldsymbol{J}_{s, 1} \boldsymbol{G}_{2} \boldsymbol{\Gamma}_{1} & \ldots & \boldsymbol{J}_{s, 1} \boldsymbol{G}_{2} \boldsymbol{\Gamma}_{p} \\
\vdots & \vdots & \vdots \\
\boldsymbol{J}_{s, p} \boldsymbol{G}_{2} \boldsymbol{\Gamma}_{1} & \ldots & \boldsymbol{J}_{s, p} \boldsymbol{G}_{2} \boldsymbol{\Gamma}_{p}
\end{array}\right]=\left[\begin{array}{ccc}
\boldsymbol{M}_{s, 1}^{-1} & \ldots & \mathbf{0} \\
\vdots & \vdots & \vdots \\
\mathbf{0} & \ldots & \boldsymbol{M}_{s, p}^{-1}
\end{array}\right],
$$

where a generated block-diagonal matrix consists of $p$ blocks and each block $i$, according to Lemma 1 , has full rank $p_{i}$, $i=1, \ldots, p$. Each off-diagonal term should be equal to zero, i.e. if $i \neq j$

$$
\boldsymbol{J}_{s, i} \boldsymbol{G}_{2} \boldsymbol{\Gamma}_{j}=\mathbf{0} .
$$

Since $\tilde{\boldsymbol{G}}_{2}=\boldsymbol{G}_{2} \boldsymbol{\Gamma}$ and (15) holds, then $\tilde{\mathcal{G}}_{j} \subset \mathcal{C}_{i}^{\perp}$ holds for each $i \neq j$, since $\tilde{\mathcal{G}}_{j} \subset \mathcal{D}_{i}^{\star}$. Then, since (13) holds, $\boldsymbol{J}_{s, i} \tilde{\boldsymbol{g}}_{j}=\mathbf{0}$ for each $i \neq j$. Furthermore, as $\boldsymbol{G} \boldsymbol{\Gamma}_{j}=\tilde{\boldsymbol{g}}_{j}$, (17) can be rewritten as

$$
\mathcal{L}_{\tilde{\boldsymbol{g}}_{j}} \boldsymbol{h}_{i}(\boldsymbol{q}, \dot{\boldsymbol{q}})=\mathbf{0}
$$

which is equivalent to the first equality of (9) and, therefore, proves the theorem.

Remark 2: It should be noted though that the choice of the matrix $\boldsymbol{\Gamma}(\boldsymbol{q})$ is not unique. Furthermore, the block-diagonal matrices $\boldsymbol{M}_{s, i}^{-1}$ of $\tilde{\boldsymbol{G}}_{s}$ can be interpreted as desired inverse inertial matrices of subtasks. In that context, the input-output noninteracting control law $\boldsymbol{\Gamma}(\boldsymbol{q})$ can be chosen so that the desired inertial behavior of the system is obtained.

Corollary 1: In the case of two subtasks

$$
\left[\begin{array}{c}
\dot{\boldsymbol{x}}_{s, 1} \\
\dot{\boldsymbol{x}}_{s, 2}
\end{array}\right]=\left[\begin{array}{l}
\boldsymbol{J}_{s, 1} \\
\boldsymbol{J}_{s, 2}
\end{array}\right] \dot{\boldsymbol{q}}
$$

the noninteraction can be achieved by

$$
\boldsymbol{\Gamma}(\boldsymbol{q})=\left[\begin{array}{ll}
\boldsymbol{N}_{s, 2}(\boldsymbol{q})^{\top} \boldsymbol{J}_{s, 1}(\boldsymbol{q})^{\top} & \boldsymbol{N}_{s, 1}(\boldsymbol{q})^{\top} \boldsymbol{J}_{s, 2}(\boldsymbol{q})^{\top}
\end{array}\right]^{\top},
$$

where $\boldsymbol{N}_{s, i}=\left(\boldsymbol{I}_{n N \times n N}-\boldsymbol{J}_{s, i}^{\# \boldsymbol{M}} \boldsymbol{J}_{s, i}\right), i=1,2$ is the nullspace projection with

$$
\boldsymbol{J}_{s, i}^{\# \boldsymbol{M}}=\boldsymbol{M}^{-1} \boldsymbol{J}_{s, i}^{\top}\left(\boldsymbol{J}_{s, i} \boldsymbol{M} \boldsymbol{J}_{s, i}^{\top}\right)^{-1}, \quad i=1,2 .
$$

Proof: Inserting (18) and (19) into (16) gives as a result a block-diagonal matrix.

\section{B. State noninteracting control}

State noninteracting control enables representation of (3) with outputs (6) in a fully decoupled form. It requires an appropriate coordinate transformation and vector $\boldsymbol{\alpha}(\boldsymbol{q}, \dot{\boldsymbol{q}})$ so that the system (3) with the applied control law (7) can be represented in new coordinates as

$$
\begin{aligned}
& \dot{\boldsymbol{x}}_{0}=\dot{\boldsymbol{x}}_{0} \\
& \ddot{\boldsymbol{x}}_{1}=\tilde{\boldsymbol{f}}_{s, 1}\left(\boldsymbol{x}_{0}, \dot{\boldsymbol{x}}_{1}\right)+\tilde{\boldsymbol{G}}_{s, 1}\left(\boldsymbol{x}_{0}\right)\left(\tilde{\boldsymbol{\tau}}_{1}^{c}+\tilde{\boldsymbol{\tau}}_{1}^{e}\right) \\
& \vdots \\
& \ddot{\boldsymbol{x}}_{p}=\tilde{\boldsymbol{f}}_{s, p}\left(\boldsymbol{x}_{0}, \dot{\boldsymbol{x}}_{p}\right)+\tilde{\boldsymbol{G}}_{s, p}\left(\boldsymbol{x}_{0}\right)\left(\tilde{\boldsymbol{\tau}}_{p}^{c}+\tilde{\boldsymbol{\tau}}_{p}^{e}\right) \\
& \boldsymbol{y}_{1}=\dot{\boldsymbol{x}}_{1} \\
& \vdots \\
& \boldsymbol{y}_{p}=\dot{\boldsymbol{x}}_{p} .
\end{aligned}
$$

We define this as state noninteraction problem.

Let us first introduce the appropriate coordinate transformation.

Proposition 2 ([6]): If the distributions $\tilde{\mathcal{D}}_{i}^{\star}, \tilde{\mathcal{D}}_{i}, \tilde{\mathcal{D}}^{\star}$ and $\tilde{\mathcal{D}}$ are nonsingular in a neighbourhood of $\left[\boldsymbol{q}_{0}^{\top}, \dot{\boldsymbol{q}}_{0}^{\top}\right]^{\top}$, there exists a neighbourhood $U_{0}$ around $\left[\boldsymbol{q}_{0}^{\top}, \dot{\boldsymbol{q}}_{0}^{\top}\right]^{\top}$ and a coordinate transformation on $U_{0}$

$$
\boldsymbol{\Phi}(\boldsymbol{q}, \dot{\boldsymbol{q}})=\left[\boldsymbol{x}_{0}, \dot{\boldsymbol{x}}_{1}, \ldots, \dot{\boldsymbol{x}}_{p}\right]^{\top}
$$

such that

$$
\begin{aligned}
& \tilde{\mathcal{D}}=\operatorname{span}\left\{\frac{\partial}{\partial \boldsymbol{x}_{0}}\right\} \\
& \mathcal{D}_{i}^{\star}=\operatorname{span}\left\{\frac{\partial}{\partial \dot{\boldsymbol{x}}_{i}}\right\}, \quad \forall i=1, \ldots, p .
\end{aligned}
$$

The coordinate transformation defines new states, termed as subtask states $\boldsymbol{\Phi}(\boldsymbol{q}, \dot{\boldsymbol{q}})=\left[\boldsymbol{x}^{\top}, \dot{\boldsymbol{x}}^{\top}\right]^{\top}$. A valid choice of the coordinate transformation is

$$
\dot{\boldsymbol{x}}_{i}=\dot{\boldsymbol{x}}_{s, i}=\boldsymbol{h}_{i}(\boldsymbol{q}, \dot{\boldsymbol{q}})=\boldsymbol{J}_{s, i}(\boldsymbol{q}) \dot{\boldsymbol{q}}, \quad \forall i=1, \ldots, p,
$$

if $\boldsymbol{J}_{s, i} \boldsymbol{J}_{s, j}^{\top}=\mathbf{0}$ for every $i \neq j$. Any other basis of $\mathcal{C}_{i}$ can be considered as the suitable projection matrix. Here we assume that (24) defines the coordinate transformation for further derivations.

In general, when a coordinate transformation is applied on (8), the state noninteraction is not immediately achieved due to the coupling effects in Coriolis and centrifugal terms [19]. More specifically, if we insert $\ddot{\boldsymbol{q}}=\boldsymbol{J}_{s}^{-1}\left(\ddot{\boldsymbol{x}}_{s}-\right.$ $\dot{\boldsymbol{J}}_{s} \boldsymbol{J}_{s}^{-1} \dot{\boldsymbol{x}}_{s}$ ) into (8), the representation of the dynamical system after the change of the coordinates is

$$
\begin{aligned}
& \ddot{\boldsymbol{x}}_{s}=\underbrace{-\boldsymbol{J}_{s} \boldsymbol{M}^{-1}\left(\boldsymbol{C} \boldsymbol{J}_{s}^{-1} \dot{\boldsymbol{x}}_{s}+\boldsymbol{\tau}^{g}\right)+\dot{\boldsymbol{J}}_{s} \boldsymbol{J}_{s}^{-1} \dot{\boldsymbol{x}}_{s}}_{\tilde{\boldsymbol{f}\left(\boldsymbol{x}_{0}, \dot{\boldsymbol{x}}_{s}\right)}} \\
& +\boldsymbol{M}_{s}^{-1} \tilde{\boldsymbol{\tau}}^{c}+\boldsymbol{M}_{s}^{-1} \boldsymbol{\Gamma}^{-1} \boldsymbol{\tau}^{e} .
\end{aligned}
$$


Representation of Coriolis and centrifugal term after the change of coordinates is therefore

$$
\boldsymbol{C}_{s}=\boldsymbol{M}_{s}\left(\boldsymbol{J}_{s} \boldsymbol{M}^{-1} \boldsymbol{C}-\boldsymbol{M}_{s} \dot{\boldsymbol{J}}_{s}\right) \boldsymbol{J}_{s}^{-1}
$$

which, in general, has off-diagonal components. Therefore, the $\tilde{\boldsymbol{f}}_{i}$ depends on $\dot{\boldsymbol{x}}_{s}$ instead of on the vector $\dot{\boldsymbol{x}}_{s, i}$ only as proposed with (21). Therefore, the state noninteraction needs to be achieved by compensating the coupling Coriolis and centrifugal terms. This can be accomplished through the control component $\boldsymbol{\alpha}(\boldsymbol{q}, \dot{\boldsymbol{q}})$ of (7). One approach would be to compensate for Coriolis, centrifugal, and gravitational terms completely, i.e. by canceling out $\boldsymbol{f}_{2}(\boldsymbol{q}, \dot{\boldsymbol{q}})$ component in (8). Instead, we design $\boldsymbol{\alpha}(\boldsymbol{q}, \dot{\boldsymbol{q}})$ which modifies Coriolis and centrifugal matrix $\tilde{\boldsymbol{C}}_{s}$ to a block-diagonal matrix $\tilde{\boldsymbol{C}}_{s}$ and compensates gravity. In this way the skew-symmetric property of $\dot{\boldsymbol{M}}_{s, i}-2 \tilde{\boldsymbol{C}}_{s, i}$, which is beneficial for passivity guarantees, is preserved in the new coordinates as well.

Theorem 2: Consider the dynamical system (3), (6) under the Assumptions 1-3 and with the applied input-output noninteracting control law (16). Then the state noninteraction problem with preserved Lagrangian dynamical structure and gravity compensation is solvable by

$$
\boldsymbol{\alpha}=\boldsymbol{M} \boldsymbol{J}_{s}^{-1} \boldsymbol{M}_{s}^{-1}\left[\begin{array}{c}
\sum_{j=2}^{p} \boldsymbol{C}_{s, 1 j} \dot{\boldsymbol{x}}_{s j} \\
\vdots \\
\sum_{j=1}^{i-1} \boldsymbol{C}_{s, i j} \dot{\boldsymbol{x}}_{s j}+\sum_{j=i+1}^{p} \boldsymbol{C}_{s, i j} \dot{\boldsymbol{x}}_{s j} \\
\vdots \\
\sum_{j=1}^{p-1} \boldsymbol{C}_{s, p j} \dot{\boldsymbol{x}}_{s j}
\end{array}\right]+\boldsymbol{\tau}^{g},
$$

with $C_{s, i j}$ being the off-diagonal matrix in row block $i$ and column block $j$.

Proof: After applying the coordinate transformation (24) and full noninteracting control law, given by (7), (16), and (26), we obtain the following set of dynamical systems

$$
\begin{aligned}
& \dot{\boldsymbol{x}}_{0}=\dot{\boldsymbol{x}}_{s}=\boldsymbol{J}_{s} \dot{\boldsymbol{q}} \\
& \ddot{\boldsymbol{x}}_{s, 1}=-\boldsymbol{M}_{s, 1}^{-1} \tilde{\boldsymbol{C}}_{s, 1} \dot{\boldsymbol{x}}_{s, 1}+\boldsymbol{M}_{s, 1}^{-1}\left(\tilde{\boldsymbol{\tau}}_{1}^{c}+\tilde{\boldsymbol{\tau}}_{1}^{e}\right) \\
& \vdots \\
& \ddot{\boldsymbol{x}}_{s, p}=-\boldsymbol{M}_{s, p}^{-1} \tilde{\boldsymbol{C}}_{s, p} \dot{\boldsymbol{x}}_{s, p}+\boldsymbol{M}_{s, p}^{-1}\left(\tilde{\boldsymbol{\tau}}_{p}^{c}+\tilde{\boldsymbol{\tau}}_{p}^{e}\right) \\
& \boldsymbol{y}_{1}=\dot{\boldsymbol{x}}_{s, 1} \\
& \vdots \\
& \boldsymbol{y}_{p}=\dot{\boldsymbol{x}}_{s, p},
\end{aligned}
$$

with $\tilde{\boldsymbol{\tau}}^{e}=\boldsymbol{\Gamma}^{-1} \boldsymbol{\tau}^{e}$. It can be observed that (27) corresponds to (21) which completes the proof.

From a practical point of view, allocating subtasks to the human operator or the robots' autonomy means ensuring that the input provided from a particular source influences only a specific subtask, while all the remaining ones are not affected by this input.

\section{PASSIVITY GUARANTEES}

The objective of this section is to propose a control approach $\tilde{\tau}_{i}^{c}, i=1, \ldots, p$ to solve Problem 2, i.e. render the subsystems in (27) passive from the inputs $\tilde{\boldsymbol{\tau}}_{i}^{e}$ to the outputs $\dot{\boldsymbol{x}}_{s, i}, \forall i=1, \ldots, p$.

The representation in (27) enables us to assign distinct control goals to the subtasks. Therefore, the control design depends on the control goal for each subtask. Typical control goals in human-robot team interaction are motion tracking or regulation if there is no interaction with the environment, and force tracking or regulation if there is contact with the environment [10]. For example, the motion of the human operator can be tracked via a motion tracking system or some other sensors, and used as a reference input. Often the control goal is to achieve desired compliant interaction dynamics, i.e. the force in contact is only limited and not explicitly tracked or regulated. We propose to use impedance control to accomplish subtask control goals and achieve passivity guarantees [10]. For that purpose, let us introduce the additional coordinate change in (27)

$$
\begin{aligned}
\boldsymbol{e}_{i} & =\boldsymbol{x}_{s, i}-\boldsymbol{x}_{s, i}^{d} \\
\dot{\boldsymbol{e}}_{i} & =\dot{\boldsymbol{x}}_{s, i}-\dot{\boldsymbol{x}}_{s, i}^{d}, \quad \forall i=1, \ldots, p
\end{aligned}
$$

where $\boldsymbol{x}_{s, i}^{d}$ is a desired setpoint or trajectory for the subtask state $\boldsymbol{x}_{s, i}$. The obtained error dynamics is

$\left[\begin{array}{c}\dot{\boldsymbol{e}}_{i} \\ \ddot{\boldsymbol{e}}_{i}\end{array}\right]=\left[\begin{array}{c}\dot{\boldsymbol{e}}_{i} \\ -\boldsymbol{M}_{s, i}^{-1} \boldsymbol{C}_{s, i}\left(\dot{\boldsymbol{e}}_{i}+\dot{\boldsymbol{x}}_{s, i}^{d}\right)\end{array}\right]+\left[\begin{array}{c}\mathbf{0} \\ \boldsymbol{M}_{s, i}^{-1}\end{array}\right]\left(\tilde{\boldsymbol{\tau}}_{i}^{c}+\tilde{\boldsymbol{\tau}}_{i}^{e}\right)-\ddot{\boldsymbol{x}}_{s, i}^{d}$,

for each subtask. The desired error dynamics can be defined in the following way

$$
\boldsymbol{M}_{s, i} \ddot{\boldsymbol{e}}_{i}+\left(\boldsymbol{C}_{s, i}+\boldsymbol{D}_{i}\right) \dot{\boldsymbol{e}}_{i}+\boldsymbol{h}_{\boldsymbol{K}, i}\left(\boldsymbol{e}_{i}\right)=\tilde{\boldsymbol{\tau}}_{i}^{e},
$$

where $i=1, \ldots, p, \boldsymbol{D}_{i}$ is a positive-definite damping matrix, and $\boldsymbol{h}_{K, i}$ is a stiffness term which shapes the desired potential energy. In order to obtain the desired error dynamics (29), it is sufficient to apply the following control law to (28)

$$
\begin{aligned}
& \tilde{\boldsymbol{\tau}}_{i}^{c}=\boldsymbol{M}_{s, i}\left(\boldsymbol{x}_{0}\right) \ddot{\boldsymbol{x}}_{s, i}^{d}+\boldsymbol{C}_{s, i}\left(\boldsymbol{x}_{0}, \dot{\boldsymbol{x}}_{s, i}\right) \dot{\boldsymbol{x}}_{s, i}^{d} \\
& -\boldsymbol{D}_{i} \dot{\boldsymbol{e}}_{i}-\boldsymbol{h}_{\boldsymbol{K}, i}\left(\boldsymbol{e}_{i}\right) .
\end{aligned}
$$

The system is passive if there exists a positive semidefinite storage function $S_{i}\left(\boldsymbol{e}_{i}, \dot{\boldsymbol{e}}_{i}\right)$ such that for all $t \geq 0$

$$
\int_{0}^{T} \dot{\boldsymbol{e}}_{i}^{\top} \tilde{\boldsymbol{\tau}}_{i}^{e} \geq S\left(\boldsymbol{e}_{i}(t), \dot{\boldsymbol{e}}_{i}(t)\right)-S\left(\boldsymbol{e}_{i}(0), \dot{\boldsymbol{e}}_{i}(0)\right)
$$

Preserving passivity in the interaction is important because it guarantees a limited transfer of energy from the robot team to the human operator or the environment. In that way, it is possible to maintain safe interactions. Let us show that the passivity is preserved with the control law (30). For that purpose we assume that the environment/human is passive.

Proposition 3: Subtask error dynamics (28) is passive from the input $\tilde{\boldsymbol{\tau}}_{i}^{e}$ to the output $\dot{\boldsymbol{e}}_{i}$ with storage function

$$
S_{i}\left(\boldsymbol{e}_{i}, \dot{\boldsymbol{e}}_{i}\right)=\frac{1}{2} \dot{\boldsymbol{e}}_{i}^{\top} \boldsymbol{M}_{s, i} \dot{\boldsymbol{e}}_{i}+\boldsymbol{V}_{K}\left(\boldsymbol{e}_{i}\right)
$$




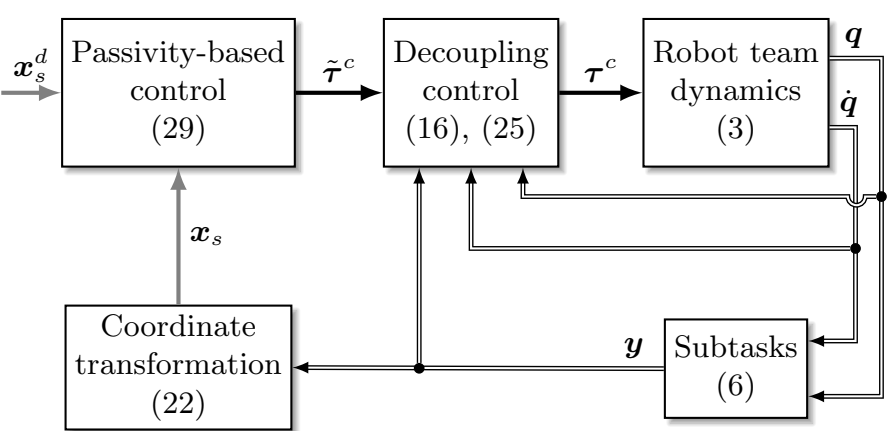

Fig. 1. Block diagram of the proposed control approach.

where $\boldsymbol{h}_{K, i}=\frac{\partial \boldsymbol{V}_{K}\left(\boldsymbol{e}_{i}\right)}{\partial \boldsymbol{e}_{i}}$ and the transformation matrix $\boldsymbol{J}_{s, i}$ is orthonormal.

Proof: Storage function (32) is an energy function and is positive semidefinite. Its derivative is

$$
\begin{aligned}
& \dot{S}_{i}=\dot{\boldsymbol{e}}_{i}^{\top} \boldsymbol{M}_{s, i} \ddot{\boldsymbol{e}}_{i}+\frac{1}{2} \dot{\boldsymbol{e}}_{i}^{\top} \dot{\boldsymbol{M}}_{s, i} \dot{\boldsymbol{e}}_{i}+\boldsymbol{h}_{K, i} \dot{\boldsymbol{e}}_{i} \\
& =\dot{\boldsymbol{e}}_{i}^{\top} \tilde{\boldsymbol{\tau}}_{i}^{e}-\dot{\boldsymbol{e}}_{i}^{\top} \boldsymbol{D}_{i} \dot{\boldsymbol{e}}_{i}+\frac{1}{2} \underbrace{\dot{\boldsymbol{e}}_{i}^{\top}\left[\dot{\boldsymbol{M}}_{s, i}-2 \boldsymbol{C}_{s, i}\right] \dot{\boldsymbol{e}}_{i}}_{=\mathbf{0}}<\dot{\boldsymbol{e}}_{i}^{\top} \tilde{\boldsymbol{\tau}}_{i}^{e} .
\end{aligned}
$$

According to (31) we conclude the system is strictly passive.

Fig. 1 depicts the block diagram of the complete control architecture.

\section{SIMULATION}

We validate the proposed control approach in a numerical simulation. Let us consider a team of 2 nonhomogeneous robots with Euler-Lagrangian dynamics.

The robots can translate in $x-y$ plane and their generalized coordinates are

$$
\boldsymbol{q}_{i}=\left[x_{i} y_{i}\right]^{\top} \in \mathbb{R}^{2} .
$$

The inertia matrix is defined as

$$
\boldsymbol{M}=\left[\begin{array}{cc}
m_{1} \boldsymbol{I}_{2} & \mathbf{0}_{2 \times 2} \\
\mathbf{0}_{2 \times 2} & m_{2} \boldsymbol{I}_{2}
\end{array}\right],
$$

with $m_{1}=1 \mathrm{~kg}$ and $m_{2}=2 \mathrm{~kg}$ being the masses of the robots. Coriolis and centrifugal, and gravitational terms are $\boldsymbol{C}=\mathbf{0}_{4 \times 4}$, and $\boldsymbol{\tau}^{g}=\mathbf{0}_{4 \times 1}$. The robot team dynamics can be rewritten as in (3)

$$
\left[\begin{array}{c}
\dot{\boldsymbol{q}} \\
\ddot{\boldsymbol{q}}
\end{array}\right]=\left[\begin{array}{c}
\dot{\boldsymbol{q}} \\
\mathbf{0}
\end{array}\right]+\left[\begin{array}{c}
\mathbf{0} \\
\boldsymbol{M}^{-1}
\end{array}\right]\left(\boldsymbol{\tau}^{c}+\boldsymbol{\tau}^{e}\right)
$$

Let us assume that the goal is that the robots maintain a formation, i.e. fixed relative distance, and move in formation in $x-y$ plane. This can be suitable for example in object transportation tasks or other formation tasks such as search and rescue.. Therefore, we define two subtasks that are the system outputs as in (6) for the described goal as

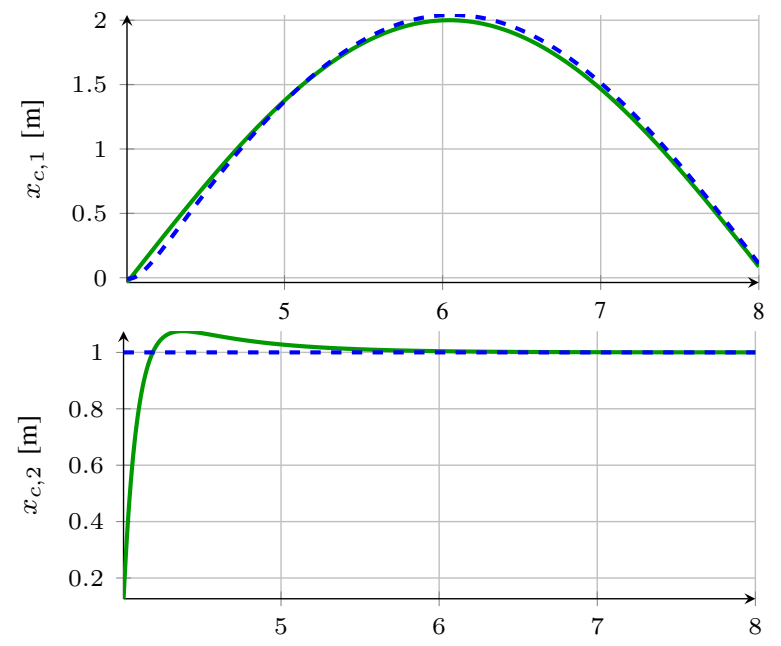

Fig. 2. Desired - - - and actual $-x$-coordinate position (up) and $y$ coordinate position (below) for the cooperative subtask.

in [10], cooperative and relative

$$
\begin{gathered}
\boldsymbol{y}_{1}=\underbrace{\frac{1}{2}\left[\boldsymbol{I}_{2} \boldsymbol{I}_{2}\right]}_{\boldsymbol{J}_{s, 1}=\boldsymbol{J}_{c}} \dot{\boldsymbol{q}} \\
\boldsymbol{y}_{2}=\underbrace{\left[\boldsymbol{I}_{2}-\boldsymbol{I}_{2}\right]}_{\boldsymbol{J}_{s, 2}=\boldsymbol{J}_{r}} \dot{\boldsymbol{q}},
\end{gathered}
$$

where $\boldsymbol{J}_{c}$ is cooperative Jacobian, and $\boldsymbol{J}_{r}$ is relative Jacobian.

We apply the noninteracting control proposed in Corollary 1 with (19) to obtain representation as in (21)

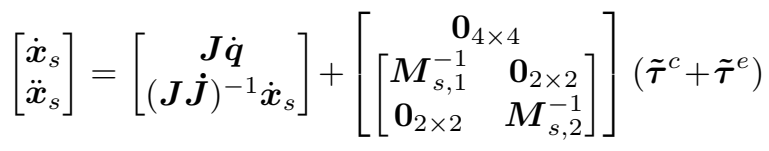

where $\boldsymbol{M}_{s, 1}^{-1}=\boldsymbol{J}_{c} \boldsymbol{M}^{-1} \boldsymbol{N}_{r}^{\top} \boldsymbol{J}_{c}^{\top}$ and $\boldsymbol{M}_{s, 2}^{-1}=$ $\boldsymbol{J}_{r} \boldsymbol{M}^{-1} \boldsymbol{N}_{c}^{\top} \boldsymbol{J}_{r}^{\top}$. Therefore, we obtain a decoupled representation for cooperative and relative dynamics. Now we can define a control law $\tilde{\boldsymbol{\tau}}^{c}$ as in (30) to obtain desired error dynamics for both subtasks as in (29). Let us assume the following control goals are posed for the cooperative and relative subtasks

$$
\begin{aligned}
& \boldsymbol{x}_{s, 1}^{d}=\boldsymbol{x}_{c}^{d}=\left[\begin{array}{ll}
2 \sin (0.79 \mathrm{t}) 1 & 1
\end{array}\right]^{\top}, \quad t \geq 4 \mathrm{~s} \\
& \boldsymbol{x}_{s, 2}^{d}=\boldsymbol{x}_{r}^{d}=\left[\begin{array}{ll}
1 & 1
\end{array}\right]^{\top}
\end{aligned}
$$

and that the controller in (30), with linear stiffness part $\boldsymbol{h}_{\boldsymbol{K}, i}\left(\boldsymbol{e}_{i}\right)=\boldsymbol{K}_{i} \boldsymbol{e}_{i}$, is used for both subtasks with $\boldsymbol{D}_{c}=$ $53 \boldsymbol{I}_{2}, \boldsymbol{K}_{c}=25 \boldsymbol{I}_{2}, \boldsymbol{D}_{r}=25 \boldsymbol{I}_{2}$, and $\boldsymbol{K}_{c}=50 \boldsymbol{I}_{2}$. We assume cooperative external torques are resulting from the grasped object dynamics, i.e. $\tilde{\boldsymbol{\tau}}_{c}^{e}=m_{o} \boldsymbol{I}_{2} \boldsymbol{v}_{c}$, with $m_{o}=1 \mathrm{~kg}$. Also, we assume relative external torques are resulting from the grasped object stiffness, i.e. $\tilde{\boldsymbol{\tau}}_{r}^{e}=k_{o} \boldsymbol{I}_{2} \boldsymbol{e}_{r}$, with $k_{o}=20$.

The $x$ and $y$ components of the desired and actual position for the cooperative subtask are depicted in Fig. 2 and for the relative subtask in Fig. 3. As can be observed, the tracking of the desired cooperative trajectory is possible. Also, it is possible to maintain the desired distance between the robots 


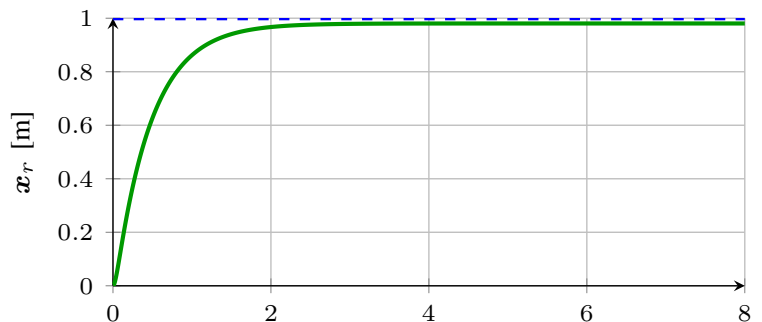

Fig. 3. Desired - - - and actual $-x$ and $y$-coordinate relative distances between the robots.

(keep the formation) without disturbances once the cooperative subtask execution starts at $t=4 \mathrm{~s}$. In practice, the human operator can provide reference cooperative trajectory through a haptic device while the robot team performs the relative subtask autonomously.

The subtasks are passive w.r.t. the storage function proposed by (32) since $\dot{M}_{s}=\mathbf{0}_{4 \times 4}$ and $\dot{\boldsymbol{J}}_{s}=\mathbf{0}_{4 \times 4}$ in (33). This can also be observed from Fig. 4 which depicts the storage functions for the two subtasks. It shows that the subsystems are passive, since the storage functions are positive semidefinite and their derivatives are negative semidefinite for all $t \geq 0$. Note that the storage function profiles and its derivatives are aligned in time for visual purposes, since the cooperative subtask is simulated to start at $t=4 \mathrm{~s}$.
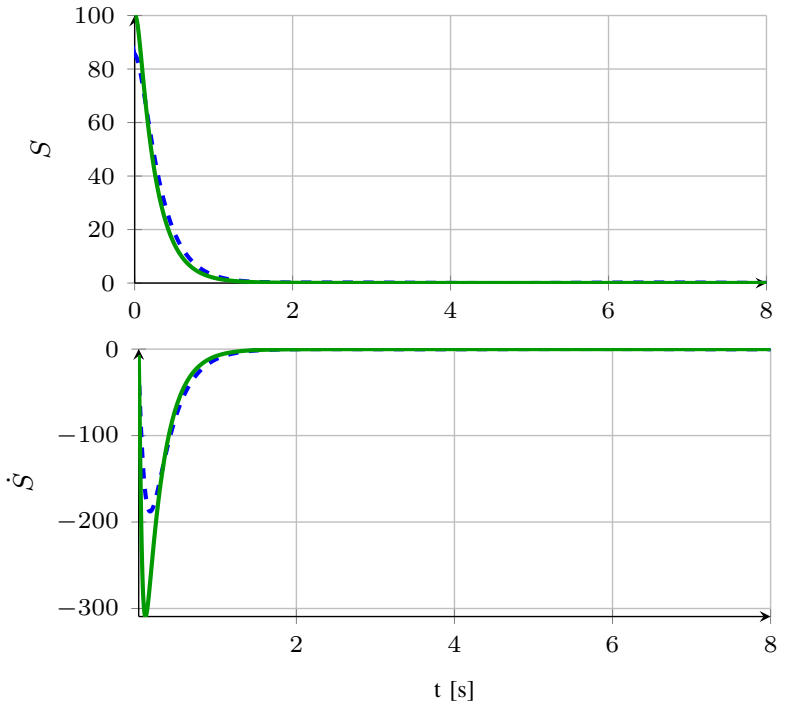

Fig. 4. Storage function for the cooperative subtask, $S_{c}---$, and the relative subtask, $S_{r}-$, (up) and their derivatives, $\dot{S}_{c}$ and $\dot{S}_{r}$, (down).

\section{CONCLUSIONS}

In this paper we propose a novel noninteracting control approach for Lagrangian systems with passivity guarantees. We show that it is possible to ensure that specific reference inputs influence specific outputs/subtasks by applying inputoutput noninteracting control. Furthermore, we show that the system dynamics can be transformed into a set of subsystems through state noninteraction control and appropriate coordinate transformation. By designing the noninteracting control law with the additional goal to preserve the Lagrangian properties of the obtained subsystems, the passivity property can be obtained. Then, each subsystem can have its own control goal. Through the simulation of a numerical example we show that it is possible to achieve control goals imposed on subtasks simultaneously.

\section{ACKNOWLEDGMENT}

This work was supported by the EU Seventh Framework Programme FP7/2007-2013 within the ERC Starting Grant Control based on Human Models (con-humo), grant agreement no. 337654.

\section{REFERENCES}

[1] D. Sieber, S. Musić, and S. Hirche, "Multi-robot manipulation controlled by a human with haptic feedback," in Intelligent Robots and Systems (IROS), 2015 IEEE/RSJ International Conference on. IEEE, 2015, pp. 2440-2446.

[2] M. Angerer, S. Musić, and S. Hirche, "Port-hamiltonian based control for human-robot team interaction," in Robotics and Automation (ICRA), 2017 IEEE International Conference on. IEEE, 2017, pp. 2292-2299.

[3] S. Musić and S. Hirche, "Control sharing in human-robot team interaction," Annual Reviews in Control, vol. 44, pp. 342-354, 2017.

[4] _ - "Classification of human-robot team interaction paradigms," in 1st IFAC Conference on Cyber-Physical \& Human-Systems, 2016, 2016.

[5] A. Isidori, Nonlinear control systems. Springer Science \& Business Media, 2013.

[6] S. Battilotti, Noninteracting control with stability for nonlinear systems. Springer-Verlag London, 1994.

[7] T. Balch and R. C. Arkin, "Behavior-based formation control for multirobot teams," IEEE Transactions on robotics and automation, vol. 14, no. 6, pp. 926-939, 1998.

[8] C. Belta and V. Kumar, "Abstraction and control for groups of robots," IEEE Transactions on robotics, vol. 20, no. 5, pp. 865-875, 2004.

[9] G. Antonelli, F. Arrichiello, and S. Chiaverini, "The null-space-based behavioral control for autonomous robotic systems," Intelligent Service Robotics, vol. 1, no. 1, pp. 27-39, 2008.

[10] S. Musić, G. Salvietti, F. Chinello, D. Prattichizzo, S. Hirche, et al., "Robot team teleoperation for cooperative manipulation using wearable haptics," in Intelligent Robots and Systems (IROS), 2017 IEEE/RSJ International Conference on. IEEE, 2017, pp. 2556-2563.

[11] D. Lee and M. W. Spong, "Bilateral teleoperation of multiple cooperative robots over delayed communication networks: Theory," in Robotics and Automation, 2005. ICRA 2005. Proceedings of the 2005 IEEE International Conference on. IEEE, 2005, pp. 360-365.

[12] M. Bonilla, L. Pallottino, and A. Bicchi, "Noninteracting constrained motion planning and control for robot manipulators," in 2017 IEEE International Conference on Robotics and Automation (ICRA), May 2017, pp. 4038-4043.

[13] J. E. Colgate, W. Wannasuphoprasit, and M. A. Peshkin, "Cobots: Robots for collaboration with human operators," in Proceedings of the 1996 ASME International Mechanical Engineering Congress and Exposition. ASME, 1996.

[14] S. Hirche and M. Buss, "Human-oriented control for haptic teleoperation," Proceedings of the IEEE, vol. 100, no. 3, pp. 623-647, 2012.

[15] S. Erhart and S. Hirche, "Model and analysis of the interaction dynamics in cooperative manipulation tasks," IEEE Transactions on Robotics, vol. 32, no. 3, pp. 672-683, 2016.

[16] H. K. Khalil, "Noninear systems," Prentice-Hall, New Jersey, vol. 2, no. 5 , pp. 5-1, 1996.

[17] A. De Luca, "Zero dynamics in robotic systems," in Nonlinear Synthesis. Springer, 1991, pp. 68-87.

[18] H. Nijmeijer and A. Van der Schaft, Nonlinear dynamical control systems. Springer, 1990, vol. 175.

[19] C. Ott, A. Dietrich, and A. Albu-Schäffer, "Prioritized multi-task compliance control of redundant manipulators," Automatica, vol. 53, pp. 416-423, 2015. 